\title{
Tetrahydrobiopterin as a Novel Therapeutic Intervention for Autism
}

\author{
Richard E. Frye, ${ }^{*}$ Lynne C. Huffman, ${ }^{\dagger \dagger}$ and Glen R. Elliott ${ }^{\dagger \dagger \S}$ \\ *Division of Child and Adolescent Neurology and The Children's Learning Institute, Department of Pediatrics, University of \\ Texas Health Science Center at Houston, Houston, Texas 77030; ${ }^{\dagger}$ The Children's Health Council, Palo Alto, California 94304; \\ ${ }^{\ddagger}$ Stanford University School of Medicine, Palo Alto, California 94305; and ${ }^{\S}$ University of California San Francisco, San \\ Francisco, California 94143
}

\begin{abstract}
Summary: Tetrahydrobiopterin $\left(\mathrm{BH}_{4}\right)$ is an essential cofactor for several critical metabolic pathways that have been reported to be abnormal in autism spectrum disorder (ASD). In addition, the cerebrospinal fluid concentration of $\mathrm{BH}_{4}$ is reported to be depressed in children with ASD. Over the past 25 years, several clinical trials have suggested that treatment with $\mathrm{BH}_{4}$ improves ASD symptomatology in some individuals. Two ongoing clinical protocols may help further define the efficacy of $\mathrm{BH}_{4}$ treatment in children with ASD. First, children with ASD who had low concentrations of cerebrospinal fluid or urine pterins were treated in an open-label manner with $20 \mathrm{mg} / \mathrm{kg}$ per day of $\mathrm{BH}_{4}$. The majority of children $(63 \%)$ responded positively to treatment, with minimal adverse events (AEs). Second, a double-blind placebo-controlled
\end{abstract}

study examining the efficacy of $20 \mathrm{mg} / \mathrm{kg}$ per day of $\mathrm{BH}_{4}$ treatment in children with ASD is currently underway. Safety studies from the commercially available forms of $\mathrm{BH}_{4}$ document the low incidence of AEs, particularly serious AEs. Studies have also documented the ability of $\mathrm{BH}_{4}$ to cross the blood-brain barrier. Based on the importance of $\mathrm{BH}_{4}$ in neurodevelopmental metabolic pathways, the safety of $\mathrm{BH}_{4}$ treatment, and the evidence for a therapeutic benefit of $\mathrm{BH}_{4}$ treatment in children with ASD, we conclude that $\mathrm{BH}_{4}$ represents a novel therapy for ASD, one that may gain wider use after further clinical studies have established efficacy and treatment guidelines. Key Words: Tetrahydrobiopterin, autism spectrum disorder, monoamine neurotransmitters, nitric oxide, treatment.

\section{INTRODUCTION}

Although many studies have clearly linked autism spectrum disorder (ASD) to CNS and systemic biological abnormalities, most autism treatment research has focused on behavioral interventions. ${ }^{1}$ Behavioral treatments are costly and labor-intensive, and may progress slowly. ${ }^{1}$ In addition, current biologically based treatments do not appear to alter the course of the disorder. ${ }^{2,3}$ Most studies of biological interventions have focused on symptom reduction, targeting the behaviors associated with ASD (e.g., self-injurious behavior, aggression, hyperactivity, and obsessive-compulsive behavior) rather than the core features of the disorder. ${ }^{3,4}$

Tetrahydrobiopterin, or $\mathrm{BH}_{4}$, is a naturally occurring substance that is an essential cofactor for several critical metabolic pathways, including those responsible for the production of monoamine neurotransmitters, the breakdown of phenylalanine, and the production of nitric ox-

Address correspondence and reprint requests to: Richard E. Frye, M.D., Ph.D., Department of Pediatrics, University of Texas Health Science Center at Houston, 7000 Fannin-UCT 2478, Houston, TX 77030. E-mail: Richard.E.Frye@uth.tmc.edu. ide. Abnormalities in several of these metabolic pathways or the products of these pathways for which $\mathrm{BH}_{4}$ is essential have been noted in individuals with ASD. In addition, abnormalities in CNS concentrations of $\mathrm{BH}_{4}$ have been reported in individuals with ASD. Over the past 25 years, a limited number of clinical trials have reported encouraging results with $\mathrm{BH}_{4}$ treatment for children with ASD. Here, we review the evidence in support of the idea that children with ASD may have a $\mathrm{BH}_{4}$ deficiency and that treatment with $\mathrm{BH}_{4}$ may be beneficial in children with ASD. In addition, we review the evidence that systemically administered $\mathrm{BH}_{4}$ actually enters the CNS, and also discuss the safety of the commercially available $\mathrm{BH}_{4}$ products that have regulatory approval.

\section{GENERAL ROLE OF $\mathrm{BH}_{4}$ IN METABOLIC SYSTEMS}

$\mathrm{BH}_{4}$ is part of the pteridine family of naturally occurring compounds with a heterocyclic pteridine structure. Pterins are pteridine derivatives with a 2-amino-4-oxo base. The pterins include $\mathrm{BH}_{4}$, biopterin, and neopterin, but only $\mathrm{BH}_{4}$ is biologically active. 


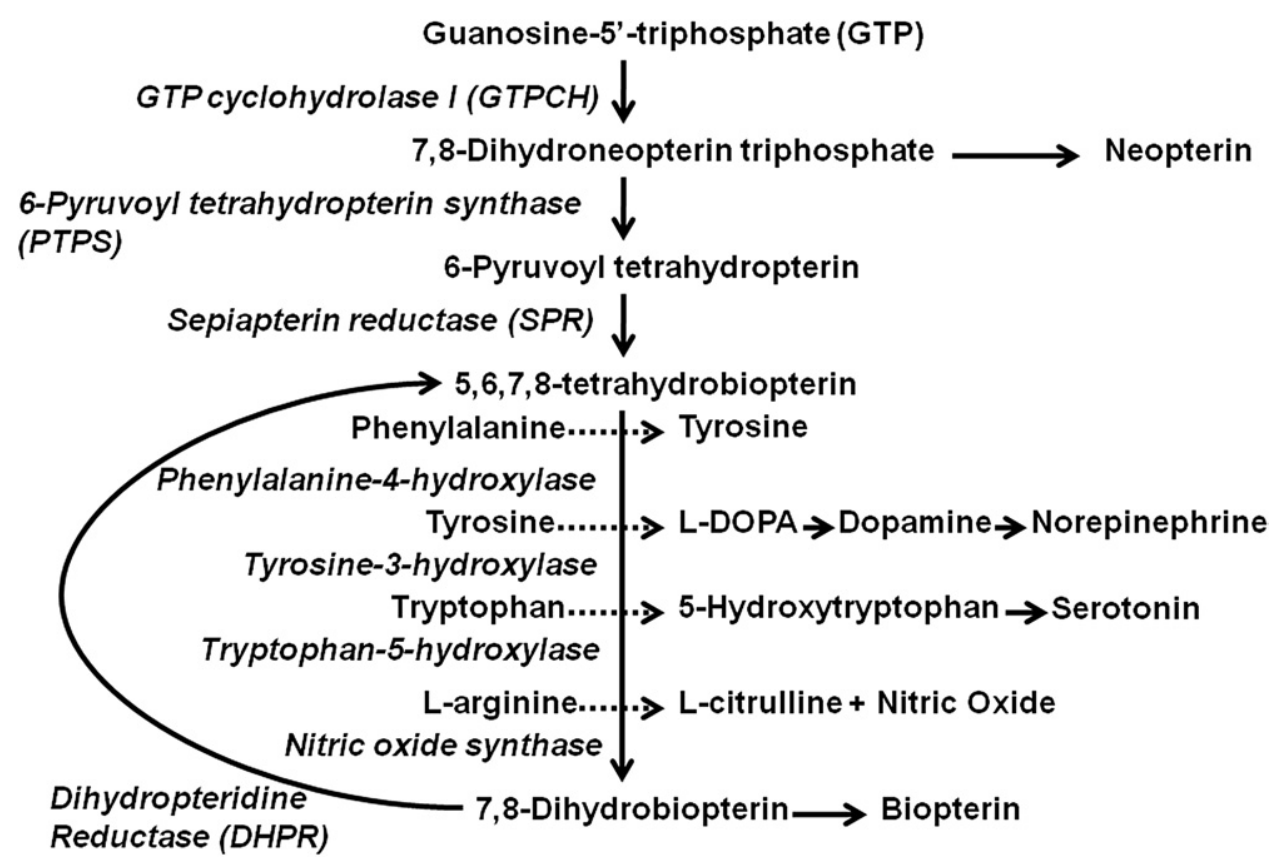

FIG. 1. Simplified pathway for the production and recycling of tetrahydrobiopterin $\left(\mathrm{BH}_{4}\right)$. Included are major enzymes, intermediates, and metabolites in this pathway. Dashed lines represent the enzymatic reactions that use $\mathrm{BH}_{4}$ to produce neurotransmitters and nitric oxide; names of enzymes are italicized.

$\mathrm{BH}_{4}$ is synthesized de novo from GTP, a purine nucleotide, by three enzymatic reactions (GTP cyclohydrolase, 6-pyruvoyltetrahydropterin synthase, and sepiapterin reductase) (FIG. 1). ${ }^{5}$ Once $\mathrm{BH}_{4}$ is synthesized, it is used as a cofactor by three aromatic amino acid hydroxylases (phenylalanine-4-hydroxylase, tyrosine-3-hydroxylase, and tryptophan-5-hydroxylase) to catalyze the conversion of phenyalanine, tyrosine, and tryptophan to tyrosine, L-dopa, and 5-hydroxytryptophan, respectively (FIG. 1). In this process, $\mathrm{BH}_{4}$ is converted into 7,8dihydrobiopterin, which can be recycled to remake $\mathrm{BH}_{4}$ through a salvage pathway catalyzed by dihydropteridine reductase. Some of these products of the hydroxylase enzymes are further converted into neurotransmitters: L-dopa is converted into dopamine, which is further converted into norepinephrine, and 5-hydroxytryptophan is converted into serotonin. Additionally, $\mathrm{BH}_{4}$ is essential in the production of nitric oxide, an important second messenger molecule used primarily for communication in vascular and neural tissues. In this reaction, nitric oxide synthase converts L-arginine and oxygen to L-citrulline and nitric oxide (FIG. 1).

A deficiency in $\mathrm{BH}_{4}$ synthesis or recycling can result in neurological disorders. Hyperphenylalaninemia, specifically phenylketonuria (PKU) type IV, presents at birth with elevated phenylalanine in the blood and represents approximately $1-2 \%$ of PKU cases. Severe mental retardation develops if phenylalanine levels are not controlled early in life. $\mathrm{BH}_{4}$ deficiency can also result in dopamine-responsive dystonia, a neurological disorder that demonstrates a wide range of symptoms and ages of presentations. Patients with dopamine-responsive dystonia invariably demonstrate dystonia with diurnal variation. ${ }^{6}$

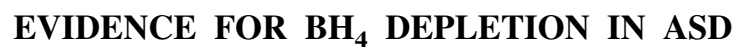

The role of $\mathrm{BH}_{4}$ in neurodevelopmental disorders, including ASD, is not well understood. The CSF concentration of $\mathrm{BH}_{4}$ has been reported to be $42 \%$ lower in children with ASD than in neurotypical children. ${ }^{7}$ Further analysis of the ASD children identified younger and older subgroups. When the subgroups were compared with the control group, only the ASD children younger than 7 years old demonstrated significantly lower $\mathrm{BH}_{4}$ concentrations. This younger group also demonstrated a reduction in total pterins and biopterin, essentially indicating that the deficit in $\mathrm{BH}_{4}$ was not due to a synthesis or recycling deficit but reflected a problem with the general availability of pterins.

Although the reason for reduction of $\mathrm{CSF} \mathrm{BH}_{4}$ in ASD is not known, there is evidence that metabolic pathways that consume and recycle $\mathrm{BH}_{4}$ are dysfunctional in children with ASD. Several lines of evidence suggest that children with ASD manifest excessive inflammation and overactivation of the immune system..$^{8-10}$ As already noted, nitric oxide, a key mediator of inflammation and immune response, is produced by a $\mathrm{BH}_{4}$-dependent reaction. Thus, it is possible that $\mathrm{BH}_{4}$ in ASD could be depleted by the overactivation of the immune system and inflammatory processes during an excessive production of nitric oxide. Children with ASD might also have an 
underlying reduction in $\mathrm{BH}_{4}$ recycling. Folate is necessary for an alternative $\mathrm{BH}_{4}$ salvage pathway the uses the enzyme dihydrofolate reductase. Available folate might be reduced in children with ASD, given that folate supports oxidative stress pathways and that the oxidative stress pathways seem to be overactive in children with ASD. ${ }^{11-14}$ Lastly, because $\mathrm{BH}_{4}$ can act as a superoxide radical scavenger, it may be depleted in the setting of high oxidative stress states. ${ }^{15,16}$

Children with ASD have symptoms not inconsistent with a $\mathrm{CNS} \mathrm{BH}_{4}$ deficiency. Such deficiency can result in low production of monoamine neurotransmitters, including serotonin, dopamine, and norepinephrine. Many children with ASD have clinical symptoms, such as obsessive-compulsive disorder and anxiety, that are seen in other disorders in which serotonin deficiency has been implicated, ${ }^{17}$ and dysfunction in the serotonin system in ASD has been documented. ${ }^{13}$ Across studies, however, response of children with ASD to selective-serotonin reuptake inhibitors has been mixed, ${ }^{18-20}$ and research suggests that at least a subgroup of children with ASD have elevations in serotonin levels. ${ }^{21}$ Children with ASD have a high rate of executive function problems, inattention, and hyperactivity, all symptoms suggesting dopamine and norepinephrine deficits. ${ }^{22-24}$ In some children with ASD, such behaviors are mitigated by psychopharmaceuticals designed to increase levels of dopamine and norepinephrine. ${ }^{25,26}$ This provides indirect evidence that some children with ASD may have deficits in the production or utilization of monoamine neurotransmitters. However, because ASD is a very heterogeneous disorder, further research will be needed to clarify the potential role of monoamine neurotransmitter deficits in ASD.

\section{PREVIOUS CLINICAL TRIALS OF $\mathrm{BH}_{4}$ FOR ASD}

In 1984, Japanese researchers reported the first case studies of $\mathrm{BH}_{4}$ treatment in children with ASD. ${ }^{27}$ Two boys with ASD, ages 7 and 5 years, were treated with 1-2.5 mg/kg per day of $\mathrm{BH}_{4}{ }^{28}$ The 7-year-old showed marked improvement in hyperactivity, mood lability, and stereotypic behaviors; both boys showed moderate improvement in general autistic symptoms. This initial evidence led to further clinical studies on $\mathrm{BH}_{4}$ as a treatment for autistic symptomatology, the majority of which were conducted in Japan. Over a 6-year period, from 1985 to 1990, four Japanese researchers studied more than 300 mildly to severely affected autistic children in five open-label studies and one double-blind placebocontrolled study. ${ }^{27-35}$ Response was measured as the percentage of children who demonstrated moderate or marked global improvement. Treatment with oral $\mathrm{BH}_{4}$ at a dose of $1-3 \mathrm{mg} / \mathrm{kg}$ per day over 4-24 weeks resulted in a response rate of $41 \%$ to $64 \%$ (Table 1). Two additional clinical studies, one double-blind placebo-controlled crossover and one open-label, were conducted by Swedish researchers using an oral $\mathrm{BH}_{4}$ dose of $3-6 \mathrm{mg} / \mathrm{kg}$ per day. ${ }^{36,37}$ These studies demonstrated limited general improvement in autistic symptoms with $\mathrm{BH}_{4}$ treatment.

Several studies have pointed to improvement in specific autistic symptoms with $\mathrm{BH}_{4}$ treatment. Nakane et al. ${ }^{34}$ noted that, within the autistic symptoms cluster of the Rating Scale for Abnormal Behavior in Children (RSABC), the greatest improvement was found in communication, cognitive ability, adaptability, and verbal expression. Fernell et al. ${ }^{37}$ found specific improvements

Table 1. Clinical Trials Evaluating Tetrahydrobiopterin $\left(\mathrm{BH}_{4}\right)$ in Autism

\begin{tabular}{|c|c|c|c|c|c|}
\hline \multirow[b]{2}{*}{ References } & \multirow[b]{2}{*}{ Tetrahydrobiopterin Treatment } & \multicolumn{2}{|c|}{ Participants, no. } & \multicolumn{2}{|c|}{$\begin{array}{l}\text { Response } \\
\text { Rate, \% }\end{array}$} \\
\hline & & $\mathrm{BH}_{4}$ & Placebo & $\mathrm{BH}_{4}$ & Placebo \\
\hline Naruse et al. ${ }^{32}(1990)$ & $\begin{array}{l}\text { Controlled Clinical Trials } \\
1-3 \mathrm{mg} / \mathrm{kg} \text { per day } \times 12 \mathrm{wk}(\text { Biopten granules).* } \\
\text { Double-blind lacebo-controlled }\end{array}$ & 41 & 42 & 53 & 31 \\
\hline Danfors et al. ${ }^{36}(2005)$ & $\begin{array}{l}6 \mathrm{mg} / \mathrm{kg} \text { per day } \times 26 \mathrm{wk}\left(\mathrm{R}_{-} \mathrm{BH}_{4}\right) \\
\text { Double-blind crossover }\end{array}$ & 12 & 0 & - & - \\
\hline & Subtotal & 53 & 42 & 53 & 31 \\
\hline $\begin{array}{l}\text { Naruse et al. }{ }^{28}(1984) \\
\text { Naruse et al. }{ }^{31}(1990) \\
\text { Takesada et al. }{ }^{35}(1990) \\
\text { Nakane et al. }{ }^{34}(1990) \\
\text { Nagahata et al }{ }^{33}(1990) \\
\text { Fernell et al. }{ }^{37}(1997) \\
\text { Subtotal } \\
\text { Grand total }\end{array}$ & $\begin{array}{l}\text { Open-Label Clinical Trials } \\
1-2.5 \mathrm{mg} / \mathrm{kg} \text { per day (Biopten granules)* } \\
1-2 \mathrm{mg} / \mathrm{kg} \text { per day } \times 4-8 \mathrm{wk} \text { (Biopten granules)* } \\
1-3 \mathrm{mg} / \mathrm{kg} \text { per day } \times 12-96 \mathrm{wk}(\text { Biopten granules)* } \\
1-3 \mathrm{mg} / \mathrm{kg} \text { per day } \times 12-105 \mathrm{wk} \text { (Biopten granules)* } \\
1-3 \mathrm{mg} / \mathrm{kg} \text { per day } \times 12-24 \mathrm{wk}(\text { Biopten granules)* } \\
3 \mathrm{mg} / \mathrm{kg} \text { per day } \times 3 \text { months }\left(\mathrm{R}^{*} \mathrm{BH}_{4}\right)^{\dagger}\end{array}$ & $\begin{array}{r}2 \\
17 \\
97 \\
24 \\
136 \\
6 \\
280 \\
333\end{array}$ & $\begin{array}{r}0 \\
0 \\
0 \\
0 \\
0 \\
0 \\
0 \\
42\end{array}$ & $\begin{array}{r}100 \\
64 \\
54 \\
42 \\
48 \\
- \\
- \\
51\end{array}$ & $\begin{array}{l}- \\
- \\
- \\
- \\
\overline{31}\end{array}$ \\
\hline
\end{tabular}

*Supplied by Daiichi Asubio Pharma (Tokyo, Japan). ${ }^{\dagger}$ Supplied by Suntory, Ltd. (Pharmaceutical Division, Tokyo, Japan). 
in social responsiveness (primarily in eye contact and social interactions), communication, and cognitive abilities using the Parental Satisfaction Survey (PASS). Danfors et al. ${ }^{36}$ demonstrated specific improvement in social interactions using the Childhood Autism Rating Scale (CARS).

Several studies have suggested that specific patient characteristics may be associated with better response to treatment with $\mathrm{BH}_{4}$. In an open-label study, Naruse et al. ${ }^{30}$ noted that younger children with ASD $(<5$ years of age) were more likely than older children with ASD ( $>10$ years of age) to respond to $\mathrm{BH}_{4}$ treatment. In a double-blind placebo-controlled study, Naruse et al. ${ }^{32}$ noted that patients under the age of 5 years had a more robust response to $\mathrm{BH}_{4}$, compared with placebo, than did those over the age of 5 years. In a double-blind placebocontrolled crossover trial, Danfors et al. ${ }^{36}$ noted that improvement in social interactions on the CARS was positively correlated with the intellectual quotient as measured by the Griffith Developmental Scales.

Although these Japanese studies treated children with-

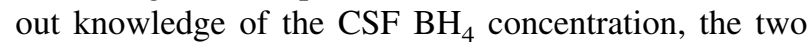
Swedish studies measured $\mathrm{CSF} \mathrm{BH}_{4}$ concentration. Fernell et al. ${ }^{37}$ and Danfors et al. ${ }^{36}$ treated children with

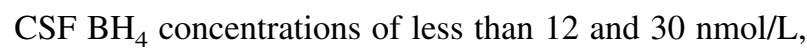
respectively. Danfors et al. ${ }^{36}$ found a borderline significant correlation $\left(r^{2}=0.25, p=0.10\right)$ between $\mathrm{CSF} \mathrm{BH}_{4}$ concentration before treatment and improvement in social interactions on the CARS with $\mathrm{BH}_{4}$ treatment. Al-

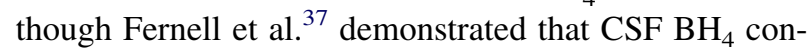
centration increased by an average of $63 \%$ with treatment, the study did not correlate initial or final CSF $\mathrm{BH}_{4}$ concentration with behavioral variables.

Only Fernell et al. ${ }^{37}$ examined functional CNS changes with treatment. With positron emission tomography, $\mathrm{BH}_{4}$ treatment was shown to decrease the baseline elevation in $\mathrm{D}_{2}$ receptor binding found in children with ASD. The authors suggested that $\mathrm{BH}_{4}$ treatment resulted in a normalization of dopamine metabolism in children with ASD.

Although the clinical trials performed to date suggest some therapeutic effect of $\mathrm{BH}_{4}$ treatment on autism symptoms, these studies are limited in several respects. First, most of the studies are open-label, leading to potential biases from the nonblinded clinicians and parents. Only two double-blind placebo-controlled studies have been performed, and one of those was limited in sample size. Larger double-blind placebo-controlled studies are needed to assess efficacy. Second, most studies have examined only behavioral markers of ASD. This may be due, in part, to the fact that the mechanism of action of $\mathrm{BH}_{4}$ in the treatment of ASD is poorly understood. It will be important to understand and measure the biological effects of $\mathrm{BH}_{4}$ treatment and understand how these effects correlate with autism symptoms. The development of such biological markers will allow clinical studies to more objectively measure the therapeutic effects of $\mathrm{BH}_{4}$ and could help improve the understanding of the biological underpinnings of ASD. Lastly, the dose of $\mathrm{BH}_{4}$ varies across studies, potentially affecting the CNS availability of $\mathrm{BH}_{4}$ in the various studies. A standard treatment protocol needs to be defined, in order to compare and combine information across trials.

\section{CURRENT TREATMENT OF ASD WITH $\mathrm{BH}_{4}$}

Currently, there are only two protocols available for the treatment of ASD with $\mathrm{BH}_{4}$. Both protocols provide $\mathrm{BH}_{4}$ in a single daily dose of $20 \mathrm{mg} / \mathrm{kg}$ per day. One of the authors (R.E.F.) is targeting children with both ASD and evidence of pterin deficiency, and the other authors (G.R.E., L.C.H.) are conducting a double-blind placebocontrolled trial examining treatment efficacy.

\section{Open-label treatment in children with ASD and pterin deficiency}

Given the importance of $\mathrm{BH}_{4}$ in critical metabolic pathways and the potential therapeutic role of $\mathrm{BH}_{4}$ in autism, patients seen in the medically based autism clinic at University of Texas Health Science Center at Houston who were diagnosed with pervasive developmental or autism disorder were screened for abnormally low pterin levels. Screening occurred after exclusion of any medical disorders that might result in neurodevelopmental disabilities, including mitochondrial, metabolic, epilepti-

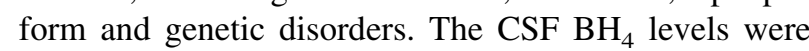
examined if available; otherwise, a fasting urine neopterin level was measured.

Patients received $\mathrm{BH}_{4}$ as sapropterin dihydrochloride (Kuvan; BioMarin, Novato, CA). Eight patients met criteria for treatment, agreed to Kuvan treatment after the benefits and risks were explained, had no contraindications for treatment with Kuvan, and provided informed consent for the examination of their clinical information for research purposes. All patients were treated with 20 $\mathrm{mg} / \mathrm{kg}$ per day of Kuvan to be taken with a meal once per day. Response to Kuvan was assessed at a clinical follow-up visit that occurred, on average, 3 months after starting Kuvan. The severity, improvement and efficacy subscales of the Clinical Global Impression Scale (CGI) were completed by the treating physician at follow-up. Severity was rated on a seven-point scale ranging from 1 , for normal, to 7, for the most severely ill patients. Improvement was rated on a seven-point scale ranging from 1 for very much improved to 7 for very much worse. The efficacy index represented clinical response versus adverse events (AEs) and ranges from 0.25 (unchanged or worsening of disease with AEs that outweigh the therapeutic effect) to 4.00 (marked improvement with no AEs). 

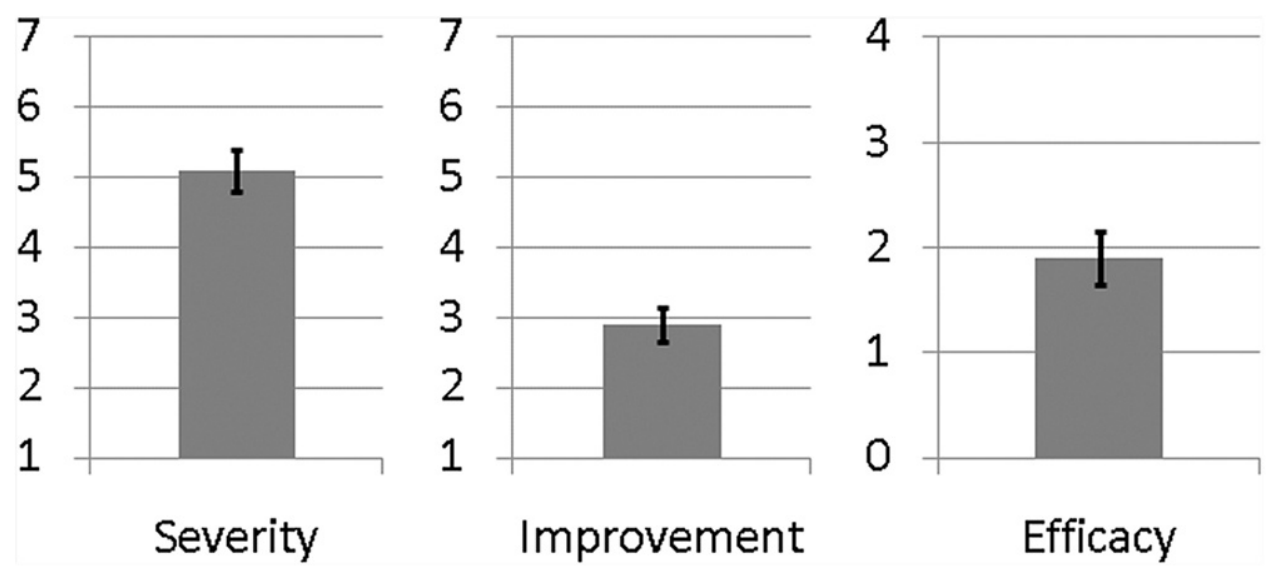

FIG. 2. The average Clinical Global Impression subscale values for eight patients with autism spectrum disorder after 3 months of oral tetrahydrobiopterin therapy at $20 \mathrm{mg} / \mathrm{kg}$ per day. On average, patients were minimally improved to much improved and had efficacy index scores representing minimal to moderate improvement with mild to no adverse events. Error bars represent standard error.

At the start of treatment, CGI severity scale scores indicated that the patients were markedly ill. Overall, five of the eight patients $(63 \%)$ responded positively to Kuvan with improvement in social interactions and either verbal or nonverbal communication. FIG. 2 presents the mean and standard error across all eight patients for the three CGI scales, as measured after Kuvan treatment. At follow-up, on average, the patients were minimally improved to much improved, with efficacy index scores representing minimal to moderate improvement and mild to no AEs.

The parents and teacher of one patient completed several behavioral questionnaires before and after $\mathrm{BH}_{4}$ supplementation. This patient demonstrated improvements in many cognitive and behavioral areas, including improvements in social awareness, communication, cognition, motivation, and autistic mannerisms on the parent and teacher Social Responsiveness Scale; lethargy and stereotypy on the parent and teacher Aberrant Behavior Checklist; and adaptive skills, depression, somatization, attention, social skills, functional communication, and activities of daily living on the parent and teacher Behavioral Assessment System for Children scale.

Five of the eight patients (63\%) demonstrated some AEs. Parents of four of the eight patients (50\%) reported mild irritability. Two of these four patients responded positively to Kuvan. In these patients, the irritability was not significant enough to discontinue or decrease the dose of Kuvan. The other two patients with irritability did not respond positively to Kuvan, and the treatment was discontinued in these patients. Notably, at 6 and 8 years of age these latter two patients were older than most of the others. One child demonstrated sleep disturbance. Decrease of the Kuvan dose to $10 \mathrm{mg} / \mathrm{kg}$ per day resulted in resolution of the sleep disturbance with continuation of the previously noted positive effects.

\section{Double-blind placebo-controlled trial with open-label extension}

At the Children's Health Council in Palo Alto, California, a phase II double-blind, placebo-controlled study randomized control trial (RCT) of sapropterin began in early 2009. Study participants are aged 3 to 6 years old, with a confirmed diagnosis of autistic disorder, and without significant cognitive delay. The primary objective of this study is to evaluate the efficacy of sapropterin (20 $\mathrm{mg} / \mathrm{kg}$ per day) versus placebo to demonstrate measureable improvements on the core symptoms of autism measured by the CGI after 16 weeks of treatment. The secondary objectives of the study are to evaluate the efficacy of sapropterin on changes in core behavioral domains and co-occurring problems such as hyperactivity, attention, and impulsiveness as indicated by direct and observational measures. In addition, the safety of sapropterin at the proposed dose in this population is being assessed, with monitoring of treatment-emergent AEs.

At the end of the 16-week randomized double-blind study, families are offered the choice of entering an open-label protocol. In this protocol, an open-label extension option is provided to subjects who completed that randomized study; those subjects are eligible for commercially formulated sapropterin (Kuvan) at 20 $\mathrm{mg} / \mathrm{kg}$ per day and they are assessed for efficacy and safety for 16 additional weeks of treatment. As in the $\mathrm{RCT}$, the primary outcome measure is the CGI, collected before, during, and after 16 weeks of treatment. Because one half of the subjects will have been exposed to placebo in the RCT study, exposing all subjects to active drug in the extension study creates the potential to see improvement in children not previously exposed to active treatment. Although this part of the study is openlabel, finding improvement primarily in children not previously exposed to sapropterin will add evidence of its 
benefits. As of writing, the study is ongoing and participant assignment (treatment $v s$ placebo) was still unknown to the investigators, so the effect of treatment could not be assessed.

\section{BH $_{4}$ : ABILITY TO ENTER THE CENTRAL NERVOUS SYSTEM}

Several animal studies have documented the ability of $\mathrm{BH}_{4}$ to cross the blood-brain barrier into the CNS. Five rhesus monkeys were administered $15-20 \mathrm{mg} / \mathrm{kg}$ of a $\mathrm{BH}_{4}$ diastereoisomer intravenously. The $\mathrm{CSF} \mathrm{BH}_{4}$ concentration increased 20- to 700-fold above baseline within 90 min of administration with peak CSF concentration occurring at 90-180 min after $\mathrm{BH}_{4}$ administration; the $\mathrm{BH}_{4}$ concentration gradually returned to baseline over 15 hours. ${ }^{38}$ Intraperitoneal administration of approximately $24 \mathrm{mg} / \mathrm{kg}$ of racemic $\mathrm{BH}_{4}$ in 12 rats increased $\mathrm{CNS} \mathrm{BH}_{4}$ concentration twofold after $90 \mathrm{~min}$ utes. ${ }^{39}$ Mice administered $\mathrm{BH}_{4}$ at $100 \mu \mathrm{mol} / \mathrm{kg}$ subcutaneously demonstrated significant increases in $\mathrm{CNS} \mathrm{BH}_{4}$ within 1-2 hours, with a significant increase in $\mathrm{CNS} \mathrm{BH}_{4}$ sustained for up to 8 hours. ${ }^{40,41}$ Oral administration of $\mathrm{BH}_{4}$ in mice has also been shown to result in an increase in the CNS activity of tyrosine hydroxylase, a $\mathrm{BH}_{4}$ dependent enzyme. ${ }^{42}$

Several case studies have examined CSF concentrations of $\mathrm{BH}_{4}$ or $\mathrm{BH}_{2}$ with oral administration of $\mathrm{BH}_{4}$ in

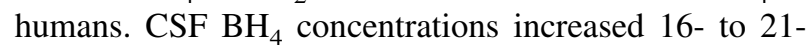
fold after six doses of racemic $\mathrm{BH}_{4}$ given at $10 \mathrm{mg} / \mathrm{kg}$ per dose every 12 hours in a 10 year-old boy with hyperphenylalaninemia. ${ }^{43} \mathrm{CSF} \mathrm{BH}_{2}$ concentration increased following $20 \mathrm{mg} / \mathrm{kg}$ of $\mathrm{BH}_{4}$ in two patients with 6-pyruvoyltetrahydropterin synthase deficiency. ${ }^{44,45}$ Fernell et

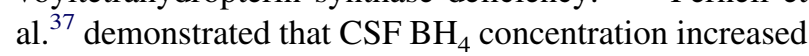
by an average of $63 \% 24$ hours after the last administration of 3 months of oral treatment with $3 \mathrm{mg} / \mathrm{kg}$ per day of $\mathrm{BH}_{4}$ divided into two daily doses. These studies provide evidence that $\mathrm{BH}_{4}$ readily crosses into the CNS in humans.

\section{SYNTHETIC $\mathrm{BH}_{4}$}

Daiichi Asubio Pharma (now Asubio Pharma in Japan, Asubio Pharmaceuticals in the USA) was the first to study the clinical use of $\mathrm{BH}_{4}$. Biopten granules $2.5 \%$ (sapropterin), a synthetic form of $\mathrm{BH}_{4}$ developed by Daiichi Asubio Pharma, was approved in Japan to treat $\mathrm{BH}_{4}$ deficiency in 1992. Kuvan brand sapropterin dihydrochloride is a synthetic preparation of the dihydrochloride salt of naturally occurring tetrahydrobiopterin $(6 R$ $\mathrm{BH}_{4}$ or $\mathrm{BH}_{4}$ ) manufactured by BioMarin (Novato, $\mathrm{CA}$ ). Kuvan has the same active ingredient as Biopten (i.e., sapropterin). Kuvan is approved by the U.S. Food and Drug Administration for $\mathrm{BH}_{4}$-responsive PKU.

\section{Clinical safety of synthetic $\mathrm{BH}_{4}$}

More than a decade of market experience with Biopten in Japan has established that it is generally safe. ${ }^{46}$ Clinical trials conducted by Daiichi Asubio Pharma and BioMarin exposed more than 1100 human participants to sapropterin formulations at various dosages. These studies indicate that sapropterin has a favorable safety profile. ${ }^{46}$ BioMarin has studied sapropterin with a dose ranging from 5 to $20 \mathrm{mg} / \mathrm{kg}$ per day. The clinical safety of Kuvan has been evaluated in several BioMarin-sponsored studies. A total of 72 healthy volunteers participated in two phase I pharmacokinetic studies, 579 subjects with PKU were treated with Kuvan in four phase II or phase III studies, and 116 poorly controlled systemic hypertension patients participated in a placebo-controlled phase II study. ${ }^{46}$

An overall analysis of the available safety data indicates that Kuvan is well tolerated and has a favorable safety profile in healthy volunteers, in subjects with PKU, and in subjects with hypertension. No deaths were reported in any of the BioMarin-sponsored studies. Adverse events reported in all studies were generally graded mild; severe AEs were infrequent. Incidence and frequency of AEs had little or no correlation with study drug dose. In placebo-controlled studies, the incidence and frequency of AEs reported for participants treated with placebo were generally comparable to those reported for subjects treated with Kuvan. The most frequently reported $(>4 \%)$ AEs were headache, diarrhea, abdominal pain, upper respiratory tract infection, pharyngolaryngeal pain, vomiting, and nausea.

In ongoing studies in children with $\mathrm{PKU}$ or $\mathrm{BH}_{4}$ deficiency, one serious adverse event, gastroesophageal reflux, was assessed by an investigator as drug-related. The participant was concomitantly taking ibuprofen, a medication known for its association with gastrointestinal disorders, and ibuprofen was reported as a second suspect medication. The physicochemical properties of $6 R$ $\mathrm{BH}_{4}$ include low $\mathrm{pH}$ and high solubility; combined, these properties could potentially increase the likelihood of developing gastrointestinal disorders, especially with the concurrent use of ibuprofen. After the event, Kuvan was reintroduced without further gastrointestinal symptoms.

In a 10 -year post-marketing safety surveillance program of sapropterin, three patients with underlying neurologic disorders experienced convulsions, exacerbation of convulsions, overstimulation, or irritability during coadministration of L-dopa and sapropterin.

Forty-three nonclinical toxicology studies have been conducted for sapropterin. ${ }^{46}$ Most of these studies were conducted in mice, rats, dogs, and marmosets at doses ranging from 20 to $4000 \mathrm{mg} / \mathrm{kg}$ using intravenous and oral administration routes. In a 52-week marmoset toxicology study, the 'no observable adverse event' level was $320 \mathrm{mg} / \mathrm{kg}$ per day. The only observed AEs were 
salivation and vomiting, both possibly caused by the low $\mathrm{pH}$ of the dosing solution; no laboratory or histopathologic abnormalities were found. In a similar 52-week rat toxicology study, the no observable $\mathrm{AE}$ level was 40 $\mathrm{mg} / \mathrm{kg}$ per day; animals treated with $400 \mathrm{mg} / \mathrm{kg}$ per day for 52 weeks demonstrated a low incidence of mild basophilic infiltrates in the renal collecting tubules; this was not associated with changes in clinical chemistry or urinalysis results. No renal tubular changes were found in a subsequent rat carcinogenicity study, in which rats were exposed to doses up to $250 \mathrm{mg} / \mathrm{kg}$ per day of sapropterin for 104 weeks. The safety of Kuvan is supported by these data and by other nonclinical pharmacology, pharmacokinetic, and toxicology studies.

\section{Clinical safety of synthetic $\mathrm{BH}_{4}$ safety in autism}

Daiichi Asubio Pharma conducted seven studies in which 451 patients with autism were treated for 5-105 weeks with sapropterin at doses of $1-5 \mathrm{mg} / \mathrm{kg}$ per day. Of the 451 patients treated, 97 (21.5\%) experienced 149 AEs for which a causal relationship with study drug could not be excluded. The most frequently reported AEs were behavioral (sleep disorders, excitement, hyperkinesias), urinary symptoms (increased frequency, enuresis, polyuria), and loose stools. There were no significant differences in the incidence of AEs between patients treated with sapropterin in all seven studies and the 115 patients treated with placebo in the two placebo-treated studies. No deaths were reported.

Four participants developed AEs severe enough to discontinue sapropterin. These AEs included hyperkinesias, panic, and tics; transient mild syncope attacks were noted in one patient with a prior history of epilepsy. For some patients, symptoms persisted for several weeks after discontinuation of sapropterin, but AEs eventually resolved in all participants. Adverse events were also reported in the Swedish clinical trials. Fernell et al. ${ }^{37}$ reported sleep disruption and increased anger and aggression in three of the six patients studied, but these symptoms were not severe enough to discontinue treatment with $\mathrm{BH}_{4}$. In the study by Danfors et al., ${ }^{36} 10$ of the 12 patients developed sleep disruption or increased aggression or both, but these AEs were just as frequent during the treatment and placebo arm of the trial. As already noted, AEs of sleep disruption and aggression were also observed in the recent series of children treated with Kuvan at University of Texas Health Science Center at Houston, but did not necessitate discontinuation of Kuvan in the children who gained benefit from the treatment.

\section{POTENTIAL FOR $\mathrm{BH}_{4}$ AS A NOVEL THERAPY IN AUTISM}

Clinical studies provide both direct and indirect evidence that the concentration of $\mathrm{CNS} \mathrm{BH}_{4}$ is depressed in children with ASD. Clinical trials suggest that treatment with $\mathrm{BH}_{4}$ results in improvement in autism symptomatology in some children with ASD. These clinical studies are mostly open-label, however, and use potentially subjective behavioral dependent measures. Two doubleblind studies have been completed, but with a limited number of participants. Further double-blind placebocontrolled studies will be needed to document further the efficacy of $\mathrm{BH}_{4}$ treatment in children with ASD. One such clinical trial currently underway should shortly provide additional information regarding the efficacy of $\mathrm{BH}_{4}$ treatment in children with ASD.

A particularly important positive characteristic of $\mathrm{BH}_{4}$ is the safety profile. $\mathrm{BH}_{4}$ has a low incidence of serious AEs and appears generally safe. In clinical trials, it has been well tolerated, and very few patients who benefit from $\mathrm{BH}_{4}$ treatment have discontinued treatment because of AEs. It is not known whether AEs are dose dependent. The treatment dose has varied widely throughout clinical studies. Further investigations may provide better insight into the optimal therapeutic dose that maximizes development and minimizes the incidence of AEs.

The importance of $\mathrm{BH}_{4}$ in ASD and other neurodevelopmental disorders should not be understated. Low CNS $\mathrm{BH}_{4}$ levels can result in devastating neurodevelopmental consequences. First, $\mathrm{BH}_{4}$ is necessary for the production of nitric oxide, a soluble molecule that is important for signaling cell proliferation, neuronal motility, and synaptic maturation during development ${ }^{47}$ and for communication between neurons and both neuronal and nonneuronal cells. ${ }^{48}$ Second, $\mathrm{BH}_{4}$ has been associated with growth factors, including nerve growth factors, in animal models. ${ }^{49}$ Third, $\mathrm{BH}_{4}$ has been shown to be a superoxide radical scavenger. ${ }^{15,16}$ Fourth, reduced levels of monoamine neurotransmitters could result in dysfunction of important neural pathways, leading to underdevelopment of such important pathways. Fifth, $\mathrm{BH}_{4}$ is an enhancer of the synaptic release of a wide range of neurotransmitters, including the catecholamines serotonin, acetylcholine, glutamate, and GABA. ${ }^{50,51}$ Thus, $\mathrm{BH}_{4}$ is involved in several metabolic and neurotransmitter pathways critical for CNS function and development. Low $\mathrm{BH}_{4}$ levels during development could have devastating consequences to the CNS, leading to or potentiating the neuropathology underlying ASD. The central role of $\mathrm{BH}_{4}$ in a wide variety of CNS and non-CNS metabolic pathways that are known to be dysfunctional in ASD makes $\mathrm{BH}_{4}$ a potentially important factor in ASD, and one that may have a therapeutic role.

\section{Disclosures}

This work was supported in part by award NS046565 (R.E.F.) and by partial salary support from an investigator-initiated clinical intervention project funded by Bio Marin, Novato, CA (L.C.H., G.R.E.). 


\section{REFERENCES}

1. Rogers SJ, Ozonoff S. Behavioral, educational, and developmental treatments of autism.. In: Moldin SO, Rubenstein JLR, editors. Understanding autism: from basic neuroscience to treatment. Boca Raton, FL: CRC Taylor \& Francis, 2006:443-473.

2. Buitelaar J. Why have drug treatments been so disappointing? In: Bock G, Goode J, editors. Autism: neural basis and treatment possibilities. New York: Novartis Foundation, 2003:235-249.

3. McDougle CJ, Posey DJ, Stigler KA. Pharmacological Treatments. In: Moldin SO, Rubenstein JLR, editors. Understanding autism: from basic neuroscience to treatment. Boca Raton, FL: CRC Taylor \& Francis, 2006:417-442.

4. Vitiello B, Wagner A. Government initiatives in autism clinical trials. CNS Spectr 2004;9:66-73.

5. Thöny B, Auerbach G, Blau N. Tetrahydrobiopterin biosynthesis, regeneration and functions. Biochem J 2000;347 Pt 1:1-16.

6. Hyland K, Surtees RA, Heales SJ, Bowron A, Howells DW, Smith I. Cerebrospinal fluid concentrations of pterins and metabolites of serotonin and dopamine in a pediatric reference population. Pediatr Res 1993;34:10-14.

7. Tani Y, Fernell E, Watanabe Y, Kanai T, Långström B. Decrease in $6 R-5,6,7,8$-tetrahydrobiopterin content in cerebrospinal fluid of autistic patients. Neurosci Lett 1994;181:169-172.

8. Dietert RR, Dietert JM. Potential for early-life immune insult including developmental immunotoxicity in autism and autism spectrum disorders: focus on critical windows of immune vulnerability. J Toxicol Environ Health B Crit Rev 2008;11:660-680.

9. Castellani ML, Conti CM, Kempuraj DJ, et al. Autism and immunity: revisited study. Int J Immunopathol Pharmacol 2009;22: 15-19.

10. Pardo CA, Vargas DL, Zimmerman AW. Immunity, neuroglia and neuroinflammation in autism. Int Rev Psychiatry 2005;17:485495.

11. James SJ, Cutler P, Melnyk S, et al. Metabolic biomarkers of increased oxidative stress and impaired methylation capacity in children with autism. Am J Clin Nutr 2004;80:1611-1617.

12. Deth R, Muratore C, Benzecry J, Power-Charnitsky VA, Waly M. How environmental and genetic factors combine to cause autism: a redox/methylation hypothesis. Neurotoxicology 2008;29:190201.

13. Pardo CA, Eberhart CG. The neurobiology of autism. Brain Pathol 2007; 17:434-447.

14. Kern JK, Jones AM. Evidence of toxicity, oxidative stress, and neuronal insult in autism. J Toxicol Environ Health B Crit Rev 2006;9:485-499.

15. Koshimura K, Murakami Y, Tanaka J, Kato Y. Self-protection of PC12 cells by $6 R$-tetrahydrobiopterin from nitric oxide toxicity. J Neurosci Res 1998;54:664-672.

16. Kojima S, Ona S, Iizuka I, Arai T, Mori H, Kubota K. Antioxidative activity of 5,6,7,8-tetrahydrobiopterin and its inhibitory effect on paraquat-induced cell toxicity in cultured rat hepatocytes. Free Radic Res 1995;23:419-430.

17. McDougle CJ, Kresch LE, Goodman WK, et al. A case-controlled study of repetitive thoughts and behavior in adults with autistic disorder and obsessive-compulsive disorder. Am J Psychiatry 1995;152:772-777.

18. Kolevzon A, Mathewson KA, Hollander E. Selective serotonin reuptake inhibitors in autism: a review of efficacy and tolerability. J Clin Psychiatry 2006;67:407-414.

19. Posey DJ, Erickson CA, Stigler KA, McDougle CJ. The use of selective serotonin reuptake inhibitors in autism and related disorders. J Child Adolesc Psychopharmacol 2006;16:181-186.

20. Soorya L, Kiarashi J, Hollander E. Psychopharmacologic interventions for repetitive behaviors in autism spectrum disorders. Child Adolesc Psychiatr Clin N Am 2008;17:753-771, viii.

21. Mulder EJ, Anderson GM, Kema IP, et al. Platelet serotonin levels in pervasive developmental disorders and mental retardation: diagnostic group differences, within-group distribution, and behavioral correlates. J Am Acad Child Adolesc Psychiatry 2004;43: 491-499.

22. Bramham J, Ambery F, Young S, et al. Executive functioning differences between adults with attention deficit hyperactivity dis- order and autistic spectrum disorder in initiation, planning and strategy formation. Autism 2009;13:245-264.

23. Corbett BA, Constantine LJ, Hendren R, Rocke D, Ozonoff S. Examining executive functioning in children with autism spectrum disorder, attention deficit hyperactivity disorder and typical development. Psychiatry Res 2009;166:210-222.

24. Jahromi LB, Kasari CL, McCracken JT, et al. Positive effects of methylphenidate on social communication and self-regulation in children with pervasive developmental disorders and hyperactivity. J Autism Dev Disord 2009;39:395-404.

25. Troost PW, Steenhuis MP, Tuynman-Qua HG, et al. Atomoxetine for attention-deficit/hyperactivity disorder symptoms in children with pervasive developmental disorders: a pilot study. J Child Adolesc Psychopharmacol 2006;16:611-619.

26. Posey DJ, Wiegand RE, Wilkerson J, Maynard M, Stigler KA, McDougle CJ. Open-label atomoxetine for attention-deficit/hyperactivity disorder symptoms associated with high-functioning pervasive developmental disorders. J Child Adolesc Psychopharmacol 2006;16:599-610.

27. Nakane Y, Naruse H, Hayashi T, Takesada M, Yamazaki K. Clinical effect of R-THBP on Infantile Autism. In: Naruse H, Ornitz E, editors. Neurobiology of infantile autism: Proceedings of the International Symposium on Neurobiology of Infantile Autism, Tokyo, 10-11 November 1990. New York: Elsevier Science Publishers, 1992:337-349.

28. Naruse H, Hayashi T, Takesada M. A preliminary study on clinical effect of tetrahydrobiopterin in infantile autism [In Japanese]. In: Reports in 1983 for New Drug Development. Tokyo: Ministry of Health and Welfare, 1984:71-81.

29. Naruse H, Hayashi T, Takesada M, Nakane Y, Yamazaki K. Metabolic changes in aromatic amino acids and monoamines in infantile autism and development of new treatment related to the findings [In Japanese]. No to Hattatu 1989;21:181-189.

30. Naruse H, Hayashi T, Takesada M, et al. Therapeutic effect of tetrahydrobiopterin in infantile autism. Proc Jpn Acad 1987;63B: 231-233.

31. Naruse H, Takesada M, Nakane Y, et al. Clinical evaluation of $R$-tetrahydrobiopterin (SUN 0588) on infantile autism: a doubleblind comparative study using placebo as a control [Japanese]. Rinsho Iyaku 1990;6:1343-1368.

32. Naruse H, Takesada M, Nagahata M, Kazamatsuri H, Nakane Y, Yamazaki K. An open clinical study of sapropterin hydrochloride ( $R$-tetrahydrobiopterin SUN 0588) in infantile autism: clinical study using a Rating Scale for Abnormal Behaviors in Children [Japanese]. Rinsho Iyaku 1990;6:1859-1875.

33. Nagahata M, Kazamatsuri H, Naruse H, et al. Clinical evaluation of sapropterin hydrochloride (R-THBP. SUN 0588) on infantile autism: a multicenter cooperative study [Japanese]. Rinsho Iyaku 1990;6:1877-1899.

34. Nakane Y, Asuo T, Shimogawa S, Fujiwara T, Kawabata Y, Kubota J. Clinical efficacy and effects on physical development of long-term treatment of $R$-tetrahydrobiopterin (R-THBP, SUN 0588) for autism[Japanese]. Kiso to Rinsho 1990;24:4579-4598.

35. Takesada M, Naruse H, Nagahata $M$. An open clinical study of sapropterin hydrochloride ( $R$-tetrahydrobiopterin, R-THBP) in infantile autism: clinical effects and long-term follow-up. In: Naruse $\mathrm{H}$, Ornitz E, editors. Neurobiology of infantile autism: Proceedings of the International Symposium on Neurobiology of Infantile Autism, Tokyo, 10-11 November 1990. New York: Elsevier Science Publishers, 1992:355-358.

36. Danfors T, von Knorring AL, Hartvig P, et al. Tetrahydrobiopterin in the treatment of children with autistic disorder: a double-blind placebo-controlled crossover study. J Clin Psychopharmacol 2005; 25:485-489.

37. Fernell E, Watanabe Y, Adolfsson I, et al. Possible effects of tetrahydrobiopterin treatment in six children with autism-clinical and positron emission tomography data: a pilot study. Dev Med Child Neurol 1997;39:313-318.

38. Miller L, Insel T, Scheinin M, et al. Tetrahydrobiopterin administration to rhesus macaques: its appearance in CSF and effect on neurotransmitter synthesis. Neurochem Res 1986;11:291-298.

39. Kapatos G, Kaufman S. Peripherally administered reduced pterins do enter the brain. Science 1981;212:955-956. 
40. Brand MP, Hyland K, Engle T, Smith I, Heales SJ. Neurochemical effects following peripheral administration of tetrahydropterin derivatives to the $h p h-1$ mouse. J Neurochem 1996;66:1150-1156.

41. Canevari L, Land JM, Clark JB, Heales SJ. Stimulation of the brain NO/cyclic GMP pathway by peripheral administration of tetrahydrobiopterin in the $h p h-1$ mouse. J Neurochem 1999;73:2563-2568.

42. Thöny B, Calvo AC, Scherer T, et al. Tetrahydrobiopterin shows chaperone activity for tyrosine hydroxylase. J Neurochem 2008; 106:672-681.

43. Kaufman S, Kapatos G, McInnes RR, Schulman JD, Rizzo WB. Use of tetrahydropterins in the treatment of hyperphenylalaninemia due to defective synthesis of tetrahydrobiopterin: evidence that peripherally administered tetrahydropterins enter the brain. Pediatrics 1982;70:376-380.

44. Ferraris S, Guardamagna O, Bonetti G, et al. Fate of peripherally administered tetrahydrobiopterin in congenital tetrahydrobiopterin deficiency. In: Curtius HC, Blau N, Levine RA, editors. Unconjugated pterins and related biogenic amines: proceedings of the first international workshop, Flims, Switzerland, February 28-March 7, 1987.New York: Walter de Gruyter, 1987:283-292.

45. al Aqeel A, Ozand PT, Gascon GG, Hughes H, Reynolds CT,
Subramanyam SB. Response of 6-pyruvoyl-tetrahydropterin synthase deficiency to tetrahydrobiopterin. J Child Neurol 1992;7 Suppl:S26-S30.

46. BioMarin Pharmaceutical Inc. Investigator's brochure. Version 4.0. Novato, CA: BioMarin Pharmaceutical, 2008.

47. Tegenge MA, Bicker G. Nitric oxide and cyclic GMP signal transduction positively regulates the motility of human neuronal precursor (NT2) cells. J Neurochem 2009;110:1828-1841.

48. Garthwaite J. Concepts of neural nitric oxide-mediated transmission. Eur J Neurosci 2008;27:2783-2802.

49. Anastasiadis PZ, Bezin L, Imerman BA, Kuhn DM, Louie MC, Levine RA. Tetrahydrobiopterin as a mediator of PC12 cell proliferation induced by EGF and NGF. Eur J Neurosci 1997;9:18311837.

50. Koshimura K, Miwa S, Lee K, Fujiwara M, Watanabe Y. Enhancement of dopamine release in vivo from the rat striatum by dialytic perfusion of $6 R$-L-erythro-5,6,7,8-tetrahydrobiopterin. J Neurochem 1990;54:1391-1397.

51. Mataga N, Imamura K, Watanabe Y. L-Threo-3,4-dihydroxyphenylserine enhanced ocular dominance plasticity in adult cats. Neurosci Lett 1992;142:115-118. 